\title{
Non-Destructive Examination Development for the JHR Material Testing Reactor
}

\author{
$\underline{\text { B. Cornu}}^{*}$, C. Roure, D. Moulin, N. Estre, D. Tisseur, M-P. Ferroud-Plattet, P. Kinnunen, P Kotiluoto, \\ A. Revuelta \\ * bernard.cornu@cea.fr
}

\begin{abstract}
The Jules Horowitz Reactor (JHR) is a European material testing reactor (MTR) under construction at the CEA Cadarache centre. It will be dedicated to material and fuel irradiation tests, as well as to the production of medical isotopes. Gamma and X-Ray benches will be implemented in the reactor pool (RER), the irradiated component storage pool (EPI) and in a shielded hot cell for measuring either the whole underwater test device still containing the experimental sample or just the experimental sample before its extraction in the hot cell.

The CEA/Cadarache Nuclear Measurement Laboratory (LMN) has been working in collaboration with VTT (Technical Research Centre in Finland Ltd.) since 2008 under a Finnish in-kind contribution agreement. This agreement focuses on the development of NDE systems implementing gamma-ray spectroscopy and high-energy $X$-ray imaging of the sample and irradiation device with the highest definition possible (resolution of $100 \mu \mathrm{m})$.
\end{abstract}

The CEA-VTT technical specifications led to a European call for tenders launched by VTT. The contract was awarded to the Spanish company IDOM for the design, manufacturing, assembly and commissioning of:

- Underwater gamma and X-ray (UGXR) mechanical benches and their associated gamma and $X$-ray collimation systems for the RER and EPI pools

- Hot cell gamma and X-ray (HGXR) bench in the JHR NDE hot cell.

The Final Design Reviews (FDR) of the UGXR and HGXR systems were completed in 2016. The design phase has been an iterative process in order to manage interfacing specifications and constraints:

- Challenging experimental requirements, mainly to cover the wide diversity of sample shapes, sample activity levels and measurement processes, but also to achieve a level of mechanical accuracy to reach the ambitious geometrical resolution target in $\mathrm{X}$-ray imaging,

- Environmental constraints (immersion, radiation, compactness, limited accessibility for maintenance),

Nuclear safety constraints (seism, radiation protection).

The whole design process has produced a number of elaborate and innovative mechatronic systems, which is rather unusual in nuclear applications since the resulting solutions have benefited from IDOM's technological expertise in designing and commissioning large telescopes for the astronomy sector.

Once the manufacturing phase and assembly finalised, the site acceptance tests for the UGXR and HGXR mechanical systems will be performed in 2019-2020 in the TOTEM facility at the CEA

Submitted for review on June 2019. This work was supported by collaboration between CEA and VTT Technical Research Centre of Finland Ldt.

B. Cornu, C. Roure, D. Moulin, N. Estre and D. Tisseur, are with CEA, DEN, Cadarache, DTN, SMTA, Nuclear Measurement Laboratory, F-13108 Saint-Paul-Lez-Durance, France. (e-mail: bernard.cornu@cea.fr).
Cadarache center. The underwater benches will be tested in the CESARINE pool to check their requirements.

\section{INTRODUCTION}

Gamma and X-Ray examinations systems (NDE) will be implemented in the future Jules Horowitz Reactor (JHR) to perform examinations on JHR experimental samples by highresolution X-ray imaging and by quantitative measurement of the spatial distribution of gamma emitters.

This article proposes a progress description of the works concerning the whole equipment for this purpose; the current state of the project now allows us to start the first acceptance test programmes for the main components.

The CEA has entrusted its Finnish partner VTT, under an inkind contribution agreement, with the design and manufacturing of the mechanical part of this equipment project. Three independent European calls for tenders were launched by VTT, with all three having been awarded to the same Spanish company, IDOM ADA.

The purpose of these contracts is to:

- Study and manufacture two identical underwater benches, called UGXR, designed to examine a JHR test device in its entirety,

- Study and manufacture a smaller bench, called HGXR, to be implemented in the hot cell for light experimental samples prior to their extraction from JHR test devices.

In parallel to the major equipment being developed under the Finnish in-kind framework contract, there is a specific project for a $6 \mathrm{MeV}$ linear accelerator whose prototypic design aims at providing a refined spot size X-ray source $(<0.5 \mathrm{~mm})$ to meet the high-resolution imaging requirement (geometrical resolution over $100 \mu \mathrm{m})$. In addition, the onsite implementation of this accelerator imposes a self-shielding design providing a ratio up to 6 decades in attenuation. The study and manufacturing of this accelerator was entrusted to the French company PMB (ALCEN Group).

$\mathrm{X}$-ray detection will be provided by a linear gamma detector based on solutions provided by ADVACAM s.r.o., a Czech company that develops detection technologies within the framework of the MEDIPIX programme at the CERN research center.

M.P. Ferroud-Plattet works at the CEA Cadarache centre also, within the DEN/JHR Reactor Service.

P. Kinnunen, P Kotiluoto and A. Revuelta work for the VTT Technical Research Centre of Finland Ldt., P.O. Box 1000 - 02044 VTT, Finland. 


\section{DESCRIPTION OF THE TWO UGXR BENCHES}

The two identical JHR UGXR underwater benches are intended to provide the following high-performance gammaray and X-ray imaging examinations, both before and after irradiation: lateral and radial scanning with high spatial resolution suitable for the wide variety of shapes and activities planned for the JHR; emission tomography; radiography; highresolution $(100 \mu \mathrm{m}) 2 \mathrm{DX}$-ray tomography; $3 \mathrm{D}$ tomography.

\section{A. Installation of both UGXR benches in the JHR}

One of these benches will be installed in the reactor pool. In addition to the fast measurements of short-lived elements afforded by such proximity to the irradiation site, this bench will also be used with particular conditions to conduct measurements on test devices for which the experimental interest is better served by maintaining the connection to the power and/or fluid supply lines during measurements.

The second UGXR bench will be installed in the EPI irradiated components storage pool and used to increase the volume of results produced per cycle. Given that this second bench will also be used outside the inter-cycle periods, it will operate in a less restricted environment, better suited to intense measurement campaigns.

Note also the significant advantage offered by these benches in terms of being able to sequence gamma and X-ray imaging measurements on both benches without the need for intermediate handling of the test device.

\section{B. General design considerations for the underwater benches}

The mechanical principle of an UGXR bench is to manoeuvre a whole JHR test device under water (using highprecision movements) in front of a collimation feed-through located at a depth of around $4 \mathrm{~m}$ that channels the gamma ray beam and the X-ray beam used for imaging. The UGXR bench must be capable of carrying a JHR test device with a maximum weight of $500 \mathrm{~kg}$ and standard overall height of $6.5 \mathrm{~m}$, the shape of which can introduce a dissymmetry of the load given that it sometimes has an off-centred top section.

In addition to the design requirements of such a large-scale, high-precision underwater mechanism, the safety requirements imposed by carrying the test device in close proximity to the core also have to be considered. Implementing all the measures required by the UGXR bench safety classification has substantially increased the workload for the manufacturer, IDOM.

To meet these extremely exacting specifications, IDOM's bench design is based on a mechanism that is almost entirely out of the water, thus essentially avoiding the issue of corrosion in water, which is particularly critical in reactor pools. The principle consists of moving an immersed arm along the $\mathrm{X}, \mathrm{Y}$ and $\mathrm{Z}$ axes and rotating it around the $\mathrm{Z}$-axis by means of a drive system located out of the water (i.e. on the upper part of the bench). The design of this exceedingly high-precision system benefits from IDOM's expertise and comprehensive operating experience in designing mechanisms used for large space observation instruments.

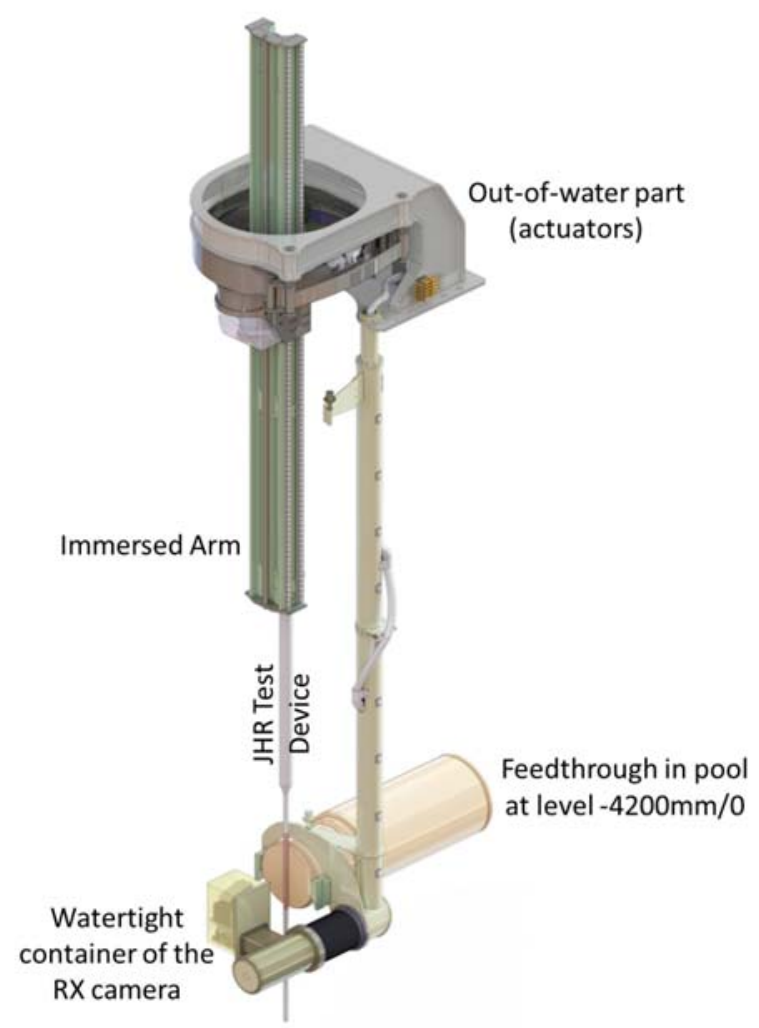

Fig. 1. Overview of the mechanism for moving an immersed JHR test device between the collimation feed-through and the submerged camera

\section{Test device gripper}

The design optimization and peak performance approach adopted by IDOM led to the test device necessarily being held at a single point, corresponding to its 'mounting plane'. To ensure the most effective means of attachment, the operator will be provided with as many different models of gripping devices as there are geometries of test devices.

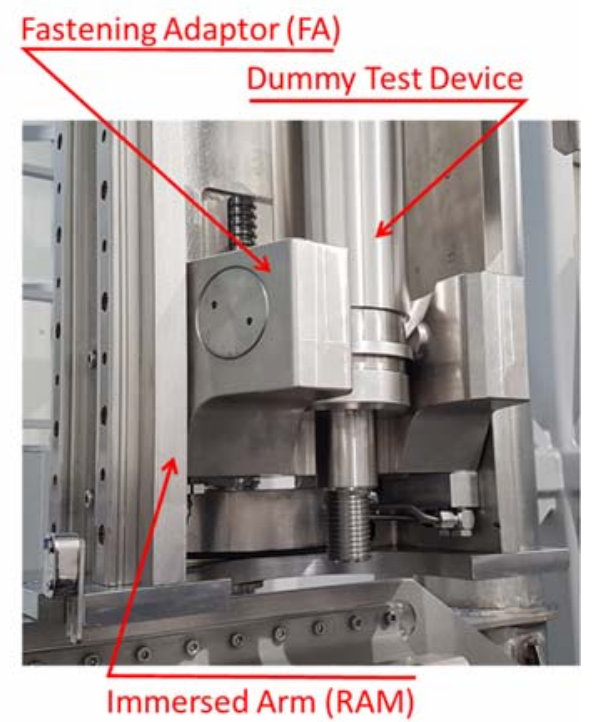

Fig. 2. View of an as-built fastening adaptor, here implemented at the factory testing site. The FA locking function is tested using a dummy test device 
The specific gripping device concerned (called a fasteningadaptor, or FA) must therefore incorporate all the fundamental safety measures. Note in particular that the mounting plane for each test device pair has a mechanical locating device ensuring the correct correspondence; and lastly that the test device is locked in position by three simultaneous positive logic locks.

\section{Specific characteristics of the rotational motion}

The X-ray tomography implemented by the UGXR benches imposes a principle of angular stability for the rotational axis, which is a key aspect of the precision sequence used to obtain high-resolution images: the purpose of a geometric resolution better than $100 \mu \mathrm{m}$ essentially dictates that the angular variation of the rotational axis falls within a an arc deviation of \pm 2 ', which represents an exceptional feat of engineering.

This level of performance can only be achieved when the centre of gravity of the load carried by the bench intercepts the axis of rotation. However, the JHR test devices carried by the bench are often unbalanced by the instrumentation mounted on their top sections. To compensate for an imbalance which is never the same for any two devices, the UGXR bench has a motorised automatic balancing mechanism designed to move two counterweights into corrective positions calculated by an algorithm based on the values supplied by three force sensors mounted on comparative supports.

Another interesting feature of the UGXR bench is the use of a push-pull drive system for the rotational motion, using a pair of opposing synchronised motors, which removes the hysteresis effects of the rotational motion by rendering them undetectable.

\section{E. Vertical stroke}

The total vertical stroke of a UGXR bench is $2880 \mathrm{~mm}$. This long stroke is used to examine a test device above the $600 \mathrm{~mm}$ height occupied by the experimental sample. For instance, the bench can use gamma spectrometry to examine the lower section of a test device that has been subjected to an accident conditions experimental sequence with gravity displacement of the fuel, immediately after an experiment. Alternatively, it can use X-ray imaging to check the conformity of an assembly of in-vessel equipment placed between the mounting plane and the experimental sample on the test device.

\section{DESCRIPTION OF UGXR X-RAY IMAGING}

The X-ray imaging principle of an UGXR bench consists of placing the test device to be examined between an accelerator placed behind the collimation feed-through and an X-ray camera placed in a built-in watertight container on the underwater bench. The following three components of the UGXR imaging system are described below: the X-ray source comprising the linear electron accelerator; the X-ray beam collimator which is one of the functions of a collimation feedthrough in the reactor pool; and the X-ray detector.

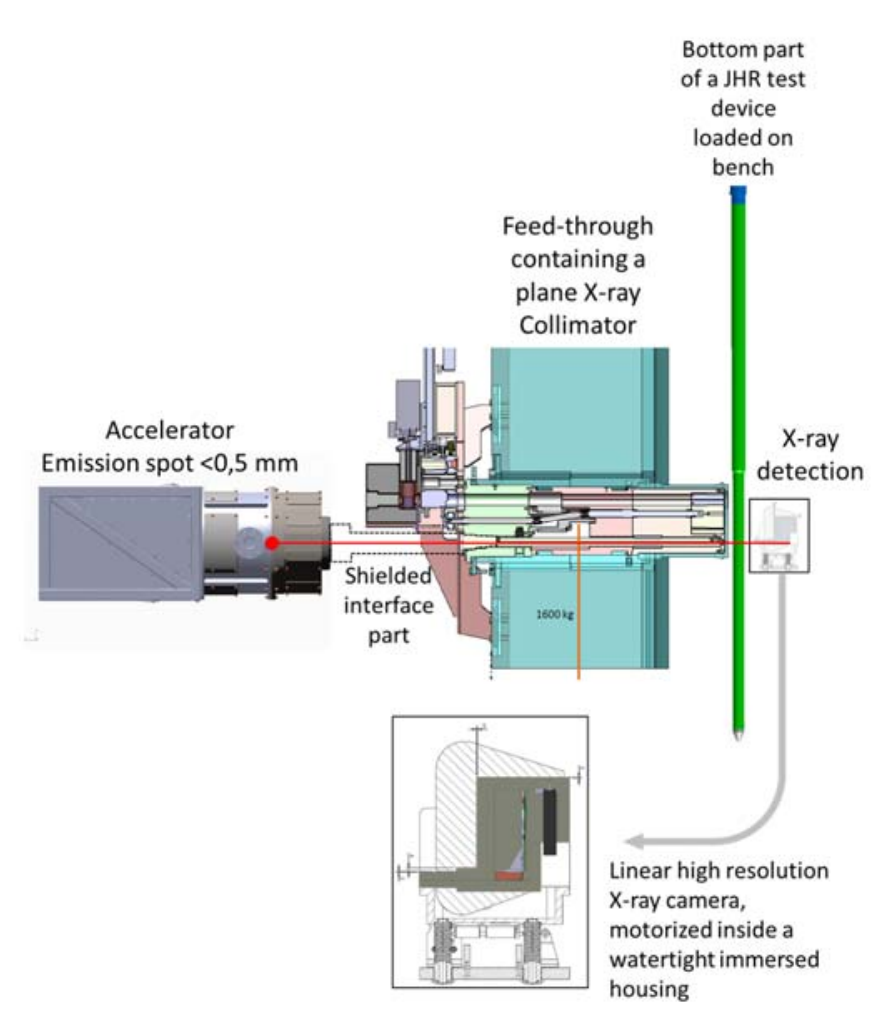

Fig. 3. Principle of X-ray imaging on the UGXR bench. The three main components are the accelerator (which provides a high-energy $\mathrm{X}$ ray source), the pool feed-through (which collimates the X-ray beam toward the object to be examined) and the X-ray detector (comprising a pixelated detection line)

\section{A. Self-shielded accelerator with smaller focal spot}

Examining the image of a JHR experimental device contained in a test device requires a high-energy X-ray beam, such as that produced by an electron accelerator.

The JHR has a single accelerator common to both UGXR benches (and the HGXR bench, see section V), which requires it to be capable of adapting to each of the three sites concerned and of being moved between sites to serve successive measurement campaigns as effectively as possible.

PMB, a subsidiary of the ALCEN group, is responsible for designing and manufacturing the accelerator. Assembly is now complete. The accelerator is currently in its testing phase. Work is ongoing to optimise its design in order to achieve the required performance levels.

The device itself is a $6 \mathrm{MeV}$ linear electron accelerator. Development of the prototype was characterised by four major specific design considerations:

- $\quad$ The focal spot needs to be less than $0.5 \mathrm{~mm}$

- The overall dimensions must comply with the highly restrictive constraints imposed by the three different installation sites

- It must be either directly or indirectly connectable to the predefined JHR pool or cell feed-throughs

- The secondary emission levels must allow the accelerator to be used in unshielded facilities

To meet this last specification, the secondary emissions emitted by the docked accelerator on the periphery must be considerably reduced by self-shielding. In order to ensure that 
the overall weight of the accelerator does not become unmanageable, the additional weight of this shielding has been optimised by incorporating the attenuating structures as close as possible to the X-ray sources that comprise the accelerator line and emission components (electron gun, acceleration line, focus triplet, target) by design.

The reduction of focal spot size required by the first specification is obtained by inserting an assembly comprising a configurable focus coil between the accelerator output and the conversion target.

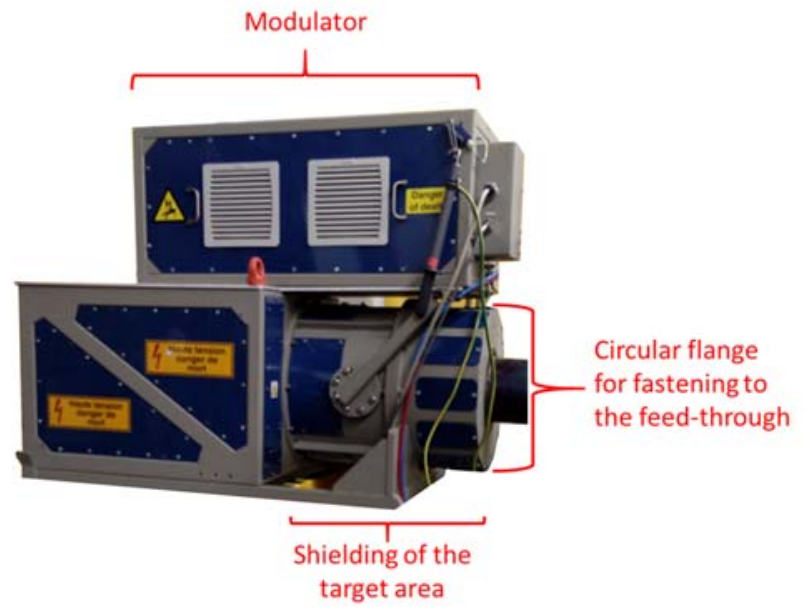

Fig. 4. View of the accelerator in the testing and optimisation phase

\section{B. X-ray beam collimator}

The JHR accelerator will emit its main beam toward the object held by the UGXR bench, through the pool wall, via a collimation feed-through to which it is docked.

For the UGXR benches it is intended for specific future contracts that a shielding-collimation interface will be created to allow continuity of the collimated X-ray beam between the accelerator and the feed-through inlet (see the interface shielding on Figure 3).

The pool feed-through X-ray collimator is around $1.5 \mathrm{~m}$ long, with a $1 \mathrm{~mm}$ planar opening. This generates a planar array beam through the experimental object that impacts the secondary collimator inlet at the submerged linear camera end.

\section{X-ray beam detection}

The principle of X-ray image formation on the UGXR benches consists of discriminating the useful X-ray flow (the localised emission source of which is targeted by a secondary collimator) from the interfering gamma flow emitted in $4 \pi$ by the emissive zone on the test device.

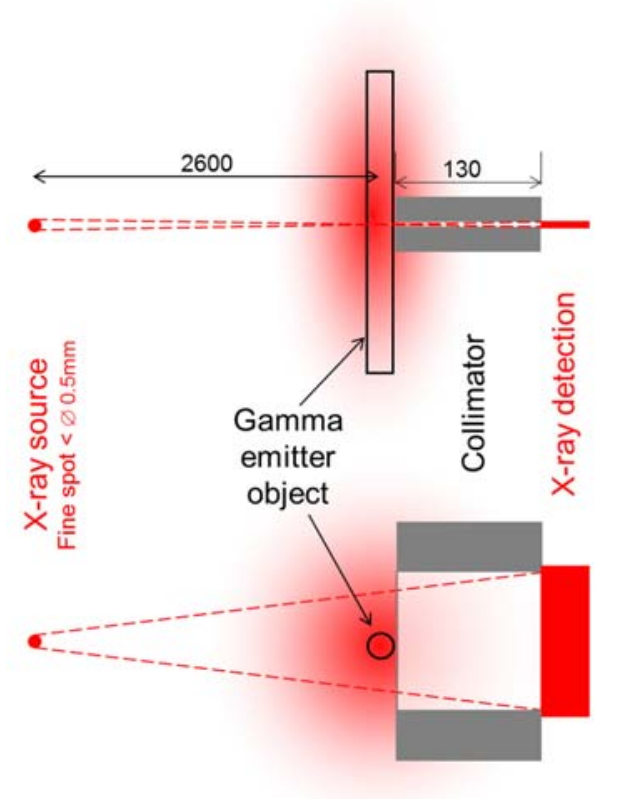

Fig. 5. Principle of linear X-ray detection adapted to a gamma emitting object

This principle is traditionally used in the nuclear industry for $\mathrm{X}$-ray/gamma ray discrimination and imposes line-by-line image acquisition, where each line corresponds to an object displacement increment between the X-ray source and the $\mathrm{X}$-ray detection line. Displacement is axial to form an X-ray; it is rotational to acquire a tomographic sinogram.

For UGXR benches, the linear X-ray camera is aligned in the array beam emitted by the accelerator. It is therefore immersed in the pool at a depth of around $4 \mathrm{~m}$ in a watertight container (see Figures 1 and 3). This container is a mechanical UGXR bench component. It is motor-driven to adapt to the varied cross-sectional dimensions of the test objects.

The camera comprises X-ray detection electronics that are shielded from radiation by a tungsten alloy housing. This heavy metal housing incorporates the discriminating secondary collimation function: a $70 \mathrm{~mm}$ wide, $130 \mathrm{~mm}$ long collimation slit with an adaptable height of $100 \mu \mathrm{m}$ or $300 \mu \mathrm{m}$ that can be used to channel the incident beam toward the high-resolution X-ray detection line downstream of the collimator. This secondary collimation function requires very precise alignment of the camera to ensure full lighting of a long collimation slit with just a $100 \mu \mathrm{m}$ opening. The I\&C system for this precision alignment function uses three piezoelectric linear actuators and a dual-axis tilt sensor. 

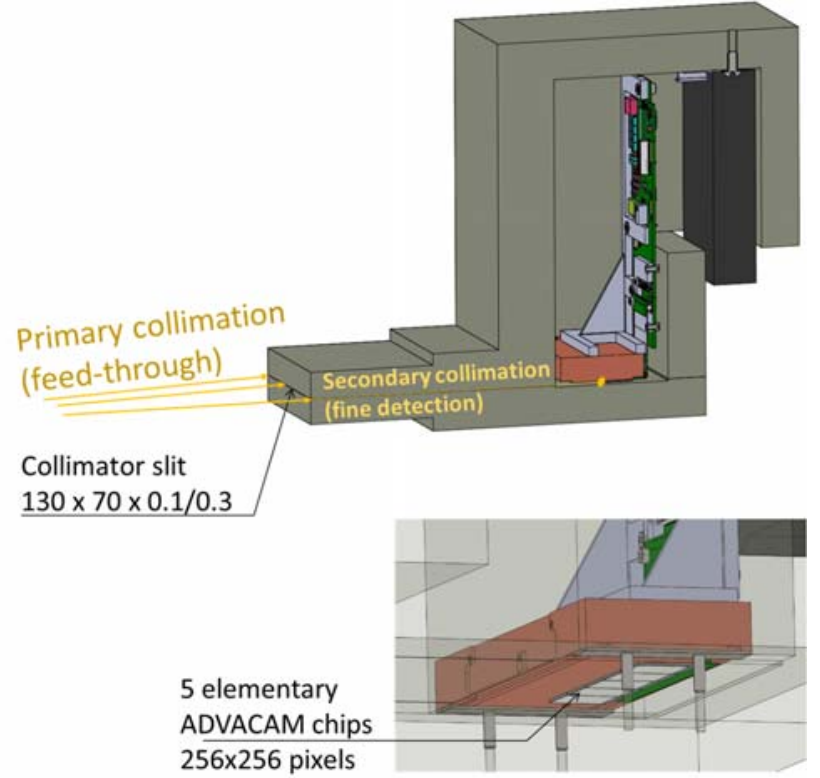

Fig. 6. Cross-section of the X-ray camera comprising a tungsten alloy shielding-collimator housing containing detection electronics based on MEDIPIX technology

The technology that allows each of the X-ray detection lines (projections) to be recorded is based on ADVACAM's WidePIX $1 \times 5$ or WidePIX $2 \times 5$ system. The principle of this system consists of aligning 5 chips, where each chip is a MEDIPIX 256x256 pixels of $55 \mu \mathrm{m}$, component with silicon semiconductor technology. This gives a whole detection made up with $256 \times 1280$ pixels, or even $512 \times 1280$ pixels if the tworow sensing arrangement is used (WidePIX $2 \times 5$ ).

As shown in Figure 6, the incident flow impacts the thin lateral edge of the 5 components line. The incident photons penetrate thus a layer of semiconducting material corresponding to a trajectory through the whole width of the components, i.e. $14 \mathrm{~mm}$ (or $28 \mathrm{~mm}$ in case of WidePIX $2 \times 5$ ). This arrangement is used to measure the spatial signal corresponding to every X-ray projection: each signal sample is reconstructed by summing the elementary signals provided by each of the pixels located on the radiation propagation line from the source.

After a series of promising parametric tests conducted by ADVACAM, it is expected that the first prototype camera will be available before the end of 2019. Its test with the accelerator is planned to take place as soon as (heavy) experimental conditions may be set up (see section VII).

\section{DESCRIPTION OF THE UGXR GAMMA MEASURING EQUIPMENT}

The gamma emitters on the test devices containing irradiated fuel can be mapped by the UGXR benches according to three degrees of freedom. This allows axial and radial gamma scanning, as well as emission tomography that combines rotation and radial scanning.

\section{A. Experimental performance of the UGXR gamma} measurements

The central requirement for the gammametry function on UGXR benches is that any existing or future gamma-emitting JHR experimental object must be measurable, given that the JHR's MTR vocation implies a wide variety of scenarios. This requirement therefore considerably extends the collimation capabilities to the following:

- Any shape of gamma emitting object: radial strokes adapted to extended shapes, like plate fuels, for instance

- All scanning modes: diversity of pre-collimation slit openings and shapes

- The widest range of gamma activities, including in particular the impact of the widest variety of fuel cooling times: diversity of post-collimation openings and screenings

The UGXR technological response to this requirement consists of:

- Fitting a pre-collimation drum made of tungsten-based material $(20 \mathrm{~cm}$ thick) inside the pool feed-through to allow the motorised interchange of 5 different types of precollimation slits. One of these slits can be interchanged manually, without having to be disassembled mechanically, in order to extend the pre-collimation mode to any other requirement not yet identified at the time the equipment was designed.

- $\quad$ Fitting a drum (15 cm thick) to the gamma-ray germanium detector to allow the motorised interchange of 6 different types of post-collimation apertures; and fitting a second drum allowing the motorised interchange of 5 calibrated copper screens ranging from 0 to $5 \mathrm{~cm}$ thick.

- Having a radial stroke of $71 \mathrm{~mm}$ on the UGXR bench to cover the majority of widths of gamma emitting objects expected in the JHR.

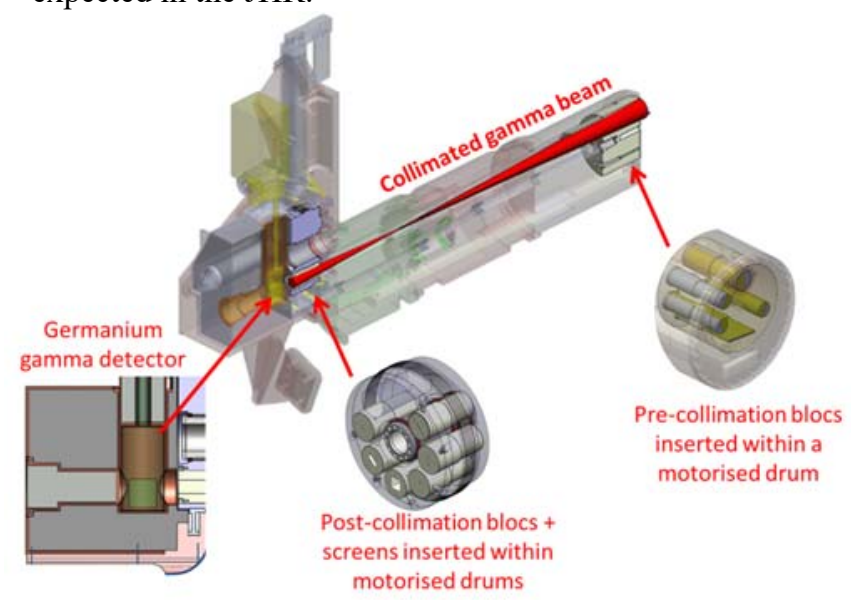

Fig. 7. Collimator-screen assembly fitted in the UGXR pool feed-through

Note that every GXR gamma-ray spectrometers use the Cryo-Pulse 5 Plus provided by Mirion Technologies, with ptype HPGe crystals (30\% efficiency), associated to LYNX systems whose Digital Signal Processing technology achieves the performance goals (energy resolution, up to the highest count rates, in particular). 


\section{B. Associated radiation protection}

The principle of collimation involves an opening which necessarily causes the local rupture of the biological shielding on the out-of-pile side, although the design source term held by the bench from the other side of the feed-through is considerable.

With regard to this specific point, the UGXR safety approach has resulted in a principle which guarantees that a radiation protection seal will be maintained in line with expectations in nominal operation or routine maintenance conditions. To demonstrate this capability, the principle uses mechanical locks and a set of authorisation keys.

The only action then needed from the classified I\&C system is the continuous display of the current status of the radiation protection in the work area adjacent to the collimation feedthrough.

\section{DESCRIPTION OF THE HGXR BENCH}

As we have seen above, both UGXR benches are intended for examining whole JHR test devices. In addition to these two benches, a smaller bench dedicated to measuring experimental loads extracted from test devices will be installed in one of the JHR's three small shielded hot cells. It offers the same experimental functions: gamma spectrometry and X-ray imaging.

\section{A. Description of the HGXR hot cell bench}

The HGXR bench, designed to measure an experimental load that has previously been extracted from its test device in a large hot cell, will support a load weighing up to $30 \mathrm{~kg}$ with a maximum height of $1200 \mathrm{~mm}$.

The HGXR equipment comprises a trolley mounted on rails on the floor of the hot cell for a recoil movement according to $(0,+2300 \mathrm{~mm})$. It is moved manually along the rails and the electrical connections are established mechanically.

This trolley has a mandrel which holds the experimental object in place. The position of the mandrel is motor-driven in terms of rotation $\left(360^{\circ}\right)$, translational movement on the y-axis $( \pm 90 \mathrm{~mm})$, and altimetry on the z-axis $(+585 \mathrm{~mm} /-1150 \mathrm{~mm})$. The trolley also accommodates the shielded camera, identical in every way to that used on the UGXR benches, except that it is not housed in a watertight container.

The design of the HGXR bench means that it may be possible to perform simple dismantling operations in the hot cell by remote handling. The conceptual design has therefore considered the constraints imposed by the clearances, the forces of the remote manipulators in the hot cell and the hanging method imposed by the manipulator grippers.

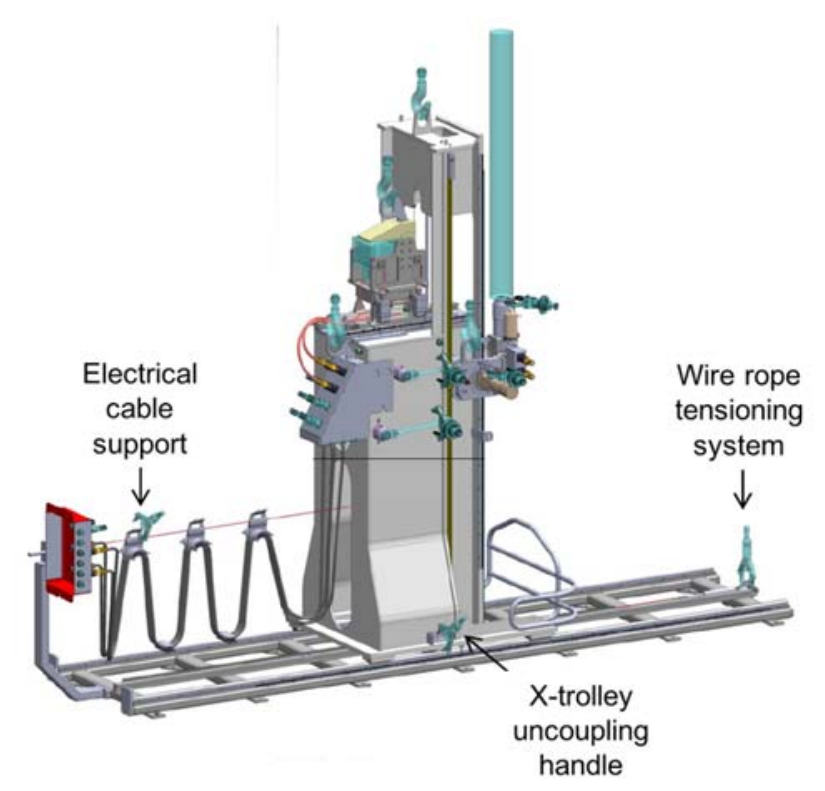

Fig. 8. Overview of the HGXR bench in the JHR ECE hot cell

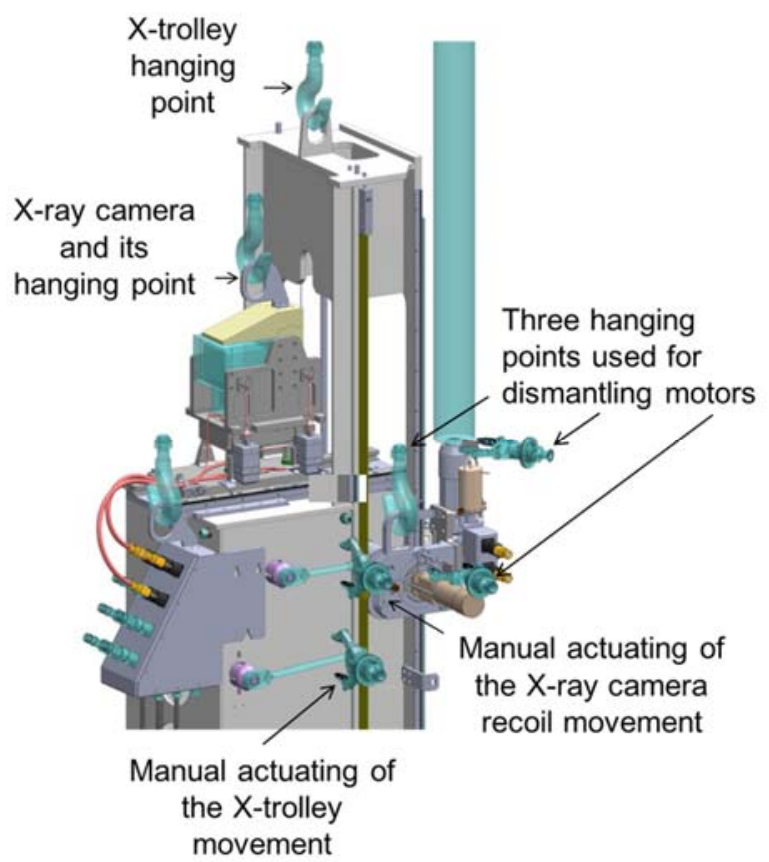

Fig. 9. Example of hanging points adapted for access by the remote manipulators and the hook on the hoisting equipment

B. Description of the HGXR equipment in the airlock adjacent to the hot cell

The principle of the HGXR bench consists of moving an experimental object in the hot cell in front of two equipped feed-throughs in the wall separating the hot cell from the adjacent airlock:

- One of the feed-throughs is dedicated to gamma measurements taken by a gamma-ray detector in the airlock, and via motorised collimation devices similar to those on the UGXR benches

- The other feed-through is dedicated to X-ray imaging via the X-ray camera on the bench, which records the X-ray 
beam generated by the accelerator in the airlock on a projection-by-projection basis.

The design is simplified by having two separate feedthroughs, in contrast to the UGXR bench.
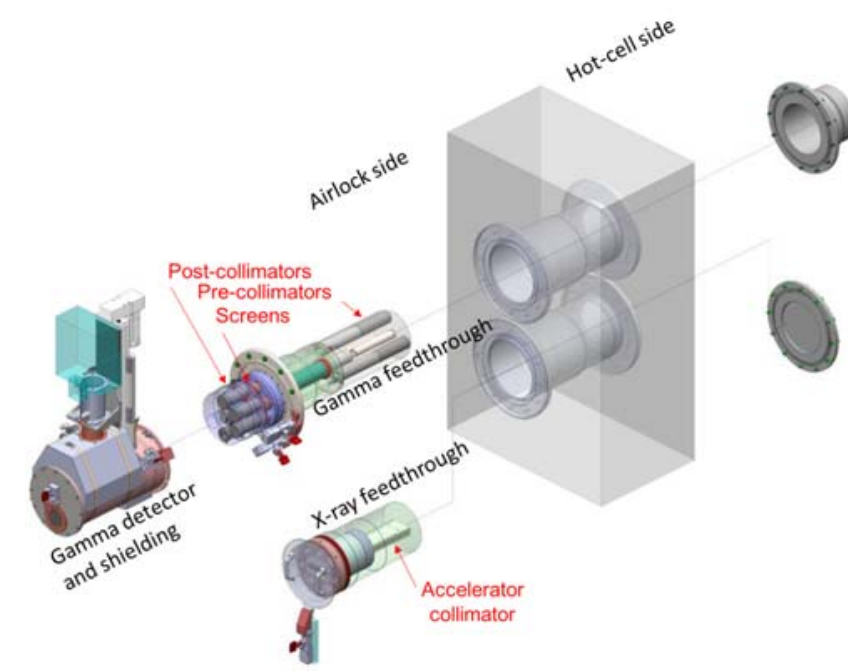

Fig. 10 .

$$
\text { measurements and X-ray imaging }
$$

The design of the collimator-screen unit for the HGXR bench is similar to that of the UGXR bench, with 3 drums which allow the interchange of 2 different pre-collimators, 4 different postcollimators and 5 different screens using a motorised system.

Similar to the UGXR, the conceptual design of the HGXR implements radiation protection measures based on locking mechanisms combined with authorisation keys.

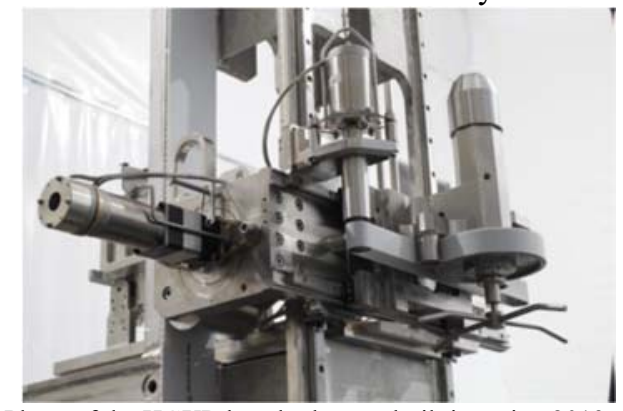

Fig. 11. Photo of the HGXR bench plate, as built in spring 2019

\section{GENERIC I\&C COMPONENTS FOR THE UGXR AND HGXR BENCHES AND THE ACCELERATOR}

With two UGXR underwater benches and one HGXR hot cell bench, there are a total of 5 GXR installation sites in the JHR. The I\&C racks for each of the two underwater benches are divided across two buildings between level 0 poolside and level -1 near the pool feed-through. The I\&C racks for the HGXR hot cell and airlock bench are located in the area in front of the small hot cells.

Each I\&C rack is equipped with a SCHNEIDER M340 PLC and associated SCADA station allowing the experimenter to monitor the equipment using specially designed tools such as mimics and alarms. There will also be up to 9 dedicated computers for experimental applications, i.e. the programmes that will be sequencing the gamma measurements (Mirion
LYNX system), X-ray measurements (ADVACAM driver), movement of experimental objects (primary PLCs), dynamic positioning of collimator-screen components (secondary PLCs), and the remote controls for the accelerator installed at any one of its three possible sites.

It will be possible to control all this equipment remotely from the JHR NDE control room. However, numerous local actions will also have to be carried out at the actual equipment locations (such as loading, settings, calibrations and maintenance). This flexible, multi-access requirement will be partially or totally done by using the BlackBox Agility KVM-over-IP remote control technology, which uses a matrix switching solution to provide a remote connection between a series of computers and a series of passive monitor-keypad-mouse stations.

\section{ACCEPTANCE PROGRAMME PLANNED AT CADARACHE}

Before being installed on-site at the JHR, all this equipment will pass through the intermediate test facilities at Cadarache. This gradual installation is necessary to adapt the schedules for the tasks being carried out by all the different stakeholders involved (IDOM, VTT, CEA) to the JHR's extremely tight schedule. It will also help in terms of implementing any tasks that need to be completed upstream to ensure the equipment is fully operational in all aspects: operating modes, safety and security, experimental procedures, maintenance, etc.

\section{A. Planned UGXR tests in the TOTEM test hall}

The majority of preparatory operations and tests will take place in the TOTEM test hall at Cadarache. This hall is therefore currently being equipped accordingly. The UGXR benches will be included in this equipment, and will be received at two additional workstations:

- $\quad$ The CESARINE pool in the TOTEM hall is equipped with a docking station to receive one of the UGXR benches that is representative of its future installation in the JHR pool. This is a fantastic opportunity to simulate the future installation of the UGXR bench and address the numerous practical issues associated with handling and maintenance operations in the calm surroundings of a dedicated hall. These operations are often difficult given that they involve manoeuvring heavy bulky objects in confined spaces.

- A test tower offers the opportunity to receive a bench that is then fully accessible to operators (full height). This second configuration is particularly suitable for running functional bench tests, including inspectable checks of safety functions, as well as all the measures required to achieve the experimental performance levels necessary for the high spatial resolution capability of these benches. 


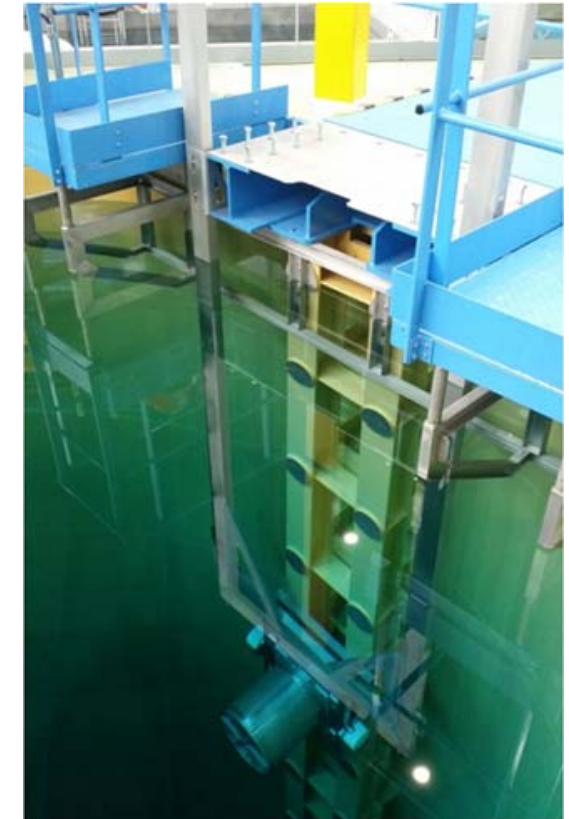

Fig. 12. Example of the equipment in the TOTEM facility: CESARINE pool equipped with docking station for the UGXR bench

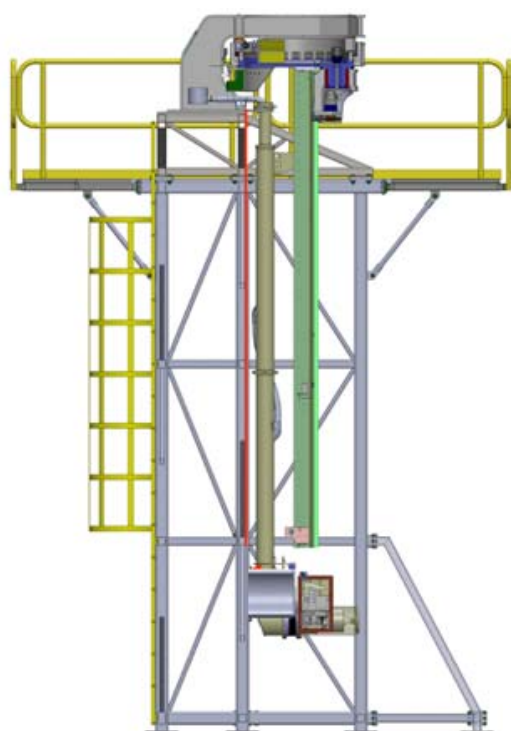

Fig. 13. Example of test equipment in the TOTEM facility: UGXR test tower

The out-of-pile parts of the collimation equipment on the UGXR benches will be placed on test stands representative of their loading in the pool feed-throughs.

To ensure that preparations are exhaustive, all the UGXR I\&C functions relating to both the in-pool and out-of-pile parts will be implemented during the tests and all preparatory operations required for the UGXR will be completed in the TOTEM facility.

The UGXR acceptance tests in TOTEM are due to start during 2020.

\section{B. Planned HGXR tests in the TOTEM test hall}

The HGXR equipment will also be received, tested and prepared in the TOTEM hall.
The HGXR test programmes are similar to many of those that will be carried out on the UGXR benches. They will be conducted in the TOTEM hall according to significantly less restrictive constraints due to the smaller component size involved.

The specific nature of the remote handling control and maintenance operations required for this hot cell bench remain the priority consideration. Studies conducted by IDOM have taken this into consideration and it has been modelled using 3D tools. However, it is only through testing in an actual hot cell mock-up like the one in the TOTEM hall that details relating to ergonomic aspects and the auxiliary tools which only become apparent at the final preparation stage can be addressed.

Getting started with the HGXR equipment in TOTEM is due to begin in the last quarter of 2019.

\section{Planned X-ray imaging tests in CINPHONIE}

The CINPHONIE test platform, located in a pit in the CHICADE facility at Cadarache, will be used to test and prepare all the X-ray imaging equipment (the ADVACAM camera and the PMB ORIATRON accelerator) before it is installed at its final destination.

Initial UGXR/HGXR camera tests and next, associated development works for UGXR measurements should be started by the end of 2019. Used with an accelerator with a smaller focal spot size and a motor-driven rotary precision indexing table, it will be tested according to a configuration that will provide a realistic simulation of the future X-ray targets on the UGXR and HGXR benches and generate an image model that will allow the predicted performance levels to be confirmed experimentally.

Regarding the JHR accelerator, it is too early for scheduling preparatory tests in CINPHONIE. A last set of outcomes related to essential optimisation works shall be prior delivered by the contractor PMB. Initial factory tests show that the smaller focal spot size of $0.5 \mathrm{~mm}$ is a viable goal. Improving the selfshielding performance, shown to be inadequate during factory tests, represents the bulk of next optimisation works.

\section{CONCLUSION}

The GXR projects, including accelerator and X-ray camera, are starting in the short term their acceptance test phases, thus concluding the industrial partners' roles.

We have seen that these testing programs will be carried out within intermediate test facilities at Cadarache, meaning that for passing from the test sites to the definitive JHR site, numerous implementing tasks, whether electrical, mechanical or dossier type, will remain still necessary for making this equipment fully functional and maintainable on site.

Furthermore, the production of experimental results at the highest level of quality initially aimed by this project will require a demanding development of software tools, whether regarding online processing tools, scientific calculation tools (including for example gamma emission tomography and 3D transmission tomography), and the secured management of any experimental data, preferentially by using a shared relational database. 